Vol.01/ No. 02

Pages: $71-80$

https://www.irojournals.com/iroiip/

DOI: https://doi.org/10.36548/jiip.2019.2.002

\title{
A NOVEL METHOD FOR HDR VIDEO ENCODING, COMPRESSION AND QUALITY EVALUATION
}

\author{
Dr. T. Senthil Kumar, \\ Associate Professor, Computer Science and Engineering Department, \\ Amrita School of Engineering, Amrita Vishwa Vidyapeetham, \\ Ettimadai P.O, Coimbatore - 641112, \\ TamilNadu, India. \\ Email: t senthilkumar@cb.amrita.edu
}

\begin{abstract}
The high dynamic range imaging/video has become more prominent in the consumer electronic realm as the it allows the human to view a picture e or a video at a minimum eye adaption. The high dynamic ranging is capable enough to capture a full range light from the scene unlike the traditional methods that captures only 8-f-stops. Though the high dynamic range video are produced using more standard methodologies, it still needs continuous developments in the distribution phase. so the paper discourses the shortcomings in the present method of high dynamic range video coding and puts forth the novel method of two layered backward compatible video encoding methods accompanied by the Chroma subsampling and the luminance masking to improve the color accuracy and remove the nonvisible portion respectively of the video content. The performance measurement of the novel method HDR video encoding compression (N-HVEC) in term of mean opinion source, bit rate savings and he HDRVQM metric is compared with the HDR10 and HDR-HLG
\end{abstract}

Keywords: High dynamic ranging, HEVC, N-HVEC, H.265, H.264, Chroma Sampling, Luminance Masking.

\section{INTRODUCTION}

The simultaneous encounters in bagging as well as signifying the real world entities with a higher constancy paves way for the necessity of the technologies with high dynamic range imaging, the capturing, storing and the displaying capability of the HDRI make them more prominent in the multimedia industries to provide an impression as well as quality that is closer to the real world appearances[1-5].

High dynamic range is specifically designed for acquiring, computing and producing back the images with the increased shadow, highlighting more details compared to the SDR video and the cinema systems competences. The high dynamic range provides more distinguishable information's that is very much closer to the visual capabilities of the human beings [6].

ISSN: 2582- 4252 
Journal of Innovative Image Processing (JIIP) (2019)

Vol.01/ No. 02

Pages: $71-80$

https://www.irojournals.com/iroiip/

DOI: https://doi.org/10.36548/jiip.2019.2.002

The word dynamic range refers to the ratio between the duskiest and the brightest parts of a scenario. As human eyes can see approximately $20-\mathrm{f}$ stops of light without any necessary adaption in the eye. The traditional or the low dynamic range or the standard dynamic range digital cameras acquire only an $8 \mathrm{f}$-stops of light that is very much less compared to the film cameras. Many methods were developed to enhance the quality of the content and HDR was developed in the Debevec and Malik' to capture the HDR images from the series of LDR exposures utilizing the knowledge of the camera response curves[7-8].

An accurately developed HDR enhances the prevailing creative palette which in turn improves the consumer experiences. Though multitudes of methods were developed to produce the high dynamic ranging video/images, the procedures supporting a qualitative transfer of the HDR video /Imaging is still in the infant stage[9].

So the paper tries to develop a novel encoding, compression methodology to overcome the short comings in the prevailing the HDR methods.

The proposed method utilizes the two layered backward compatible video encoding method accompanied by the Chroma sub sampling and the luminance masking for the HDR video content to improve the quality, preserve the luminance and evade the nonvisible portions in the content.

The remaining paper is arranged with the 2. Providing the particulars of the existing system, 3. Providing the proposed novel method for HDR video content, encoding, compression, 4. Providing the result evaluation and 5. The conclusion of the paper followed by references.

\section{EXISTING METHODS}

There are many current techniques that support the distribution of the high dynamic range video, although the information's of the HDR are acquired using the more standardized methods such as the HLG (Hybrid Log Gamma) or the PQ (Perceptual Quantizer), The HDR video distribution is still in the beginning stage. The fig .1 provides the particulars of the various coding techniques available for the high dynamic range.

ISSN: 2582- 4252 
Journal of Innovative Image Processing (JIIP) (2019)

Vol.01/ No. 02

Pages: $71-80$

https://www.irojournals.com/iroiip/

DOI: https://doi.org/10.36548/jiip.2019.2.002

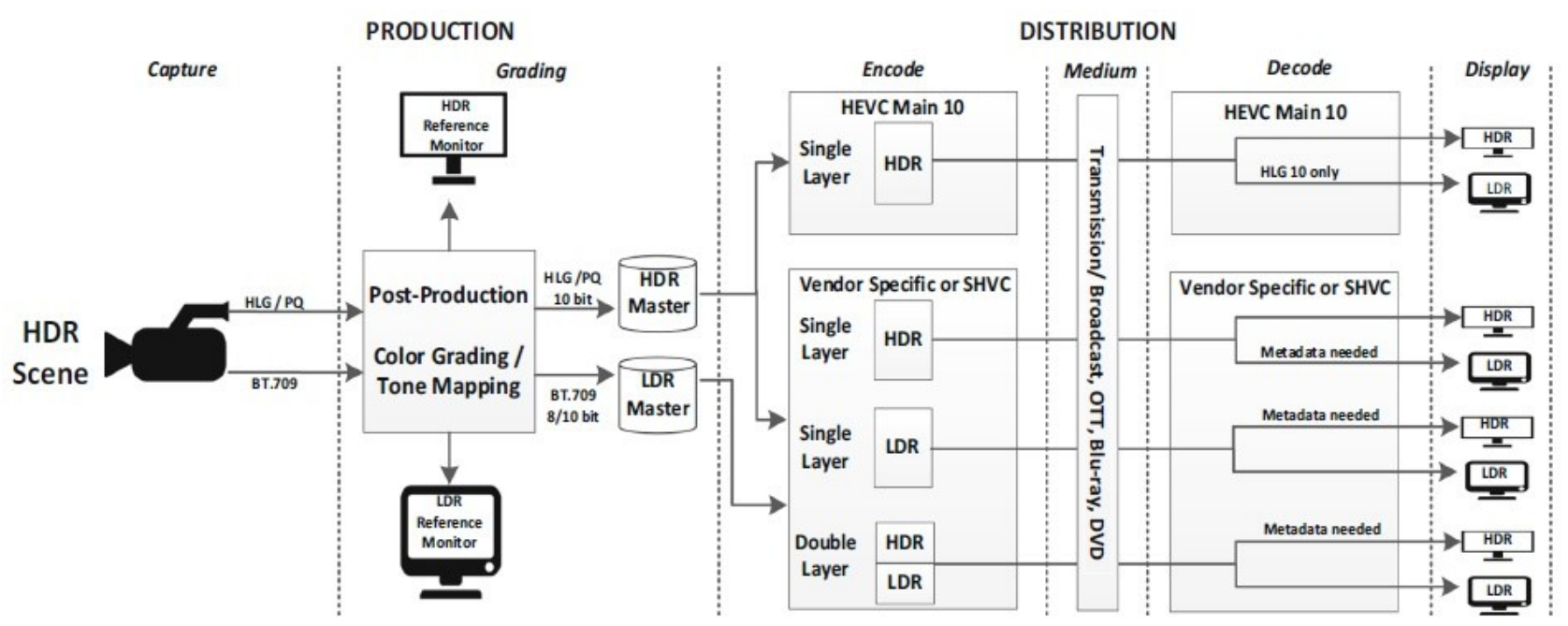

Fig. 1 HDR coding Technique [10]

Some of the existing coding techniques for the high dynamic range video content are basically categorized as the back ward compatible and non-back ward compatible methods some of the table.1 below presents some of the coding techniques available. 
Journal of Innovative Image Processing (JIIP) (2019)

Vol.01/ No. 02

Pages: $71-80$

https://www.irojournals.com/iroiip/

DOI: https://doi.org/10.36548/jiip.2019.2.002

\begin{tabular}{|c|c|c|}
\hline Methods & Transfer Function utilized & Procedure \\
\hline Double layer [11] & Utilizes LOG Transfer function & $\begin{array}{l}\text { Encodes information with similar } \\
\text { characteristics as LDR content }\end{array}$ \\
\hline Double layer residual method [12] & Utilizes PQ transfer function & $\begin{array}{l}\text { Utilizes an adaptive non linear } \\
\text { mapping function - }\end{array}$ \\
\hline Double Layer residual method [13] & employs PU-CIE transfer function & $\begin{array}{l}\text { An advanced filtering model based } \\
\text { on the HVS attributes, reduces the } \\
\text { bit rate of the residual stream }\end{array}$ \\
\hline One layer [14] & LOGLuv transfer function & $\begin{array}{l}\text { Uses Lloyd max algorithm ,Noise } \\
\text { filtering is done by employing the } \\
\text { HVS model in the discrete wavelet } \\
\text { transform domain }\end{array}$ \\
\hline One layer $[15]$ & GsLog & $\begin{array}{l}\text { Uses single codec instance and the } \\
\text { existing HDR design }\end{array}$ \\
\hline One layer $[16]$ & PQ & $\begin{array}{l}\text { PQ curve is future proof being able } \\
\text { to store very large luminance range } \\
\text { far exceeding the capabilities of } \\
\text { current displays }\end{array}$ \\
\hline One layer [17] & HLG & $\begin{array}{l}\text { No metadata is required. Works on } \\
\text { relative luminance values therefore } \\
\text { are independent of the target } \\
\text { display. Backward compatibility } \\
\text { using } \\
\text { single-layer }\end{array}$ \\
\hline
\end{tabular}

Table.1 Existing HDR CODEC Methods

\section{NOVEL ENCODING COMPRESSION TECHNIQUE}


Journal of Innovative Image Processing (JIIP) (2019)

Vol.01/ No. 02

Pages: $71-80$

https://www.irojournals.com/iroiip/

DOI: https://doi.org/10.36548/jiip.2019.2.002

The proposed method in the paper puts forth the novel technique to improve the quality of the HDR video distribution with limited bandwidth utilization. By employing the double layer backward residua method that employs the PQ [12] transfer function and further employs the Chroma subsampling [18] to preserve the luminance of the video and applies the luminance masking [5] to remove the unwanted non-visible portions in the video. The figure. 2 below shows the block diagram of the proposed methodology.

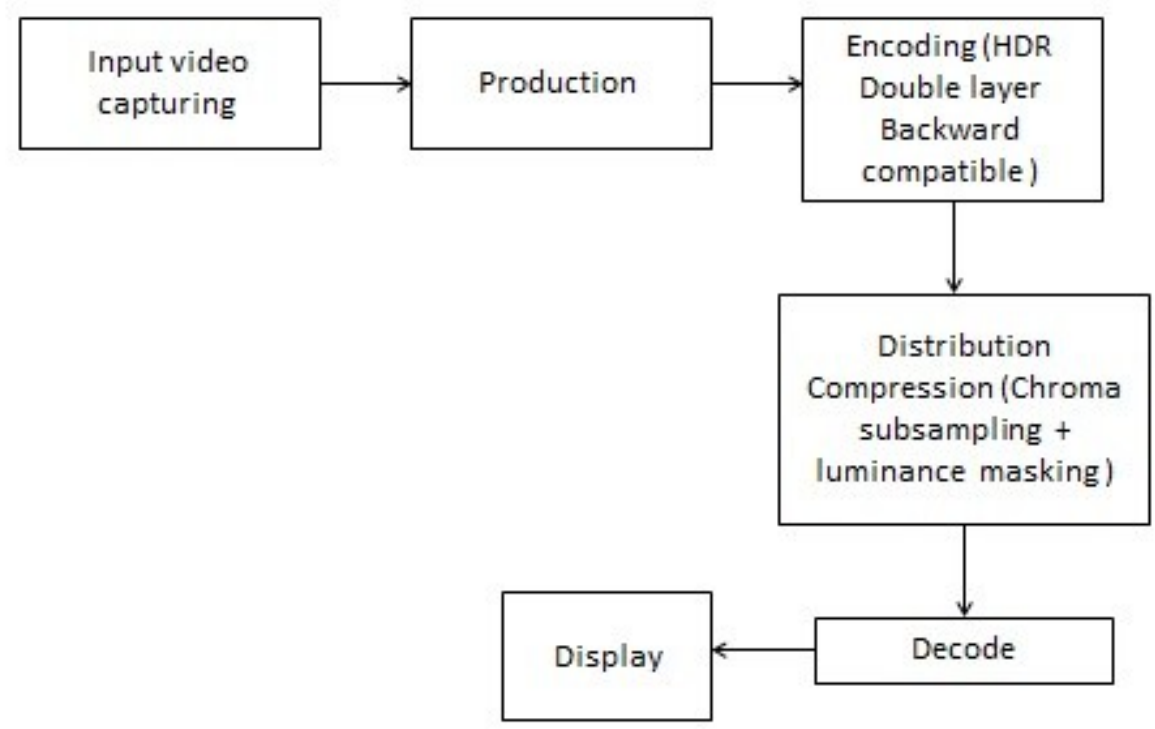

Fig.2 Proposed Method

\subsection{DOUBLE LAYER BACKWARD-COMPATIBLE ENCODING [12]}

The double layer backward compatible method for the high dynamic ranging video encoding, utilizes the instances with the dual codes in a double lane scalable manner to ensure the required compatibility between the high dynamic ranging and the low dynamic ranging for the distribution. The base layer is the termed as the first layer, offers the low dynamic range to retain the suitableness along with the decoders and the LDR displays. The high dynamic range video produces the LDR content through the tone mapping or the color mapping. The next layer does the encoding process it encodes the difference that prevails between the LDR generated and the HDR representations. The encoded data is conveyed in the quantized residual form. The reconstruction function utilizes correlation between 
Journal of Innovative Image Processing (JIIP) (2019)

Vol.01/ No. 02

Pages: $71-80$

https://www.irojournals.com/iroiip/

DOI: https://doi.org/10.36548/jiip.2019.2.002

the LDR and the HDR to predict the HDR using the LDR. The double layer backward-compatible encoding are much advantages compared to the single layer backward compatibility that uses the metadata and inverts the tone mapping curve to produce the LDR content from the HDR representations, these solution highly rely on the tradeoff between the amount of information required and the quality of the reconstructed content.

\subsection{DISTRIBUTION OF HIGH DYNAMIC RANGING [18][5]}

(i).Chroma Sub Sampling: The color information of the video occupies a separate space in the video, the color information and the space it occupies are directly proportional, the more the color information exists the more space it will take. So a simple way to reduce the color information in the video is to compress the color information or discard some of them this is called the Chroma subsampling the subsampling is achieved by transforming the red, blue and the green spectrum into the $\mathrm{YC}_{B} \mathrm{C}_{R}$, where the ' $\mathrm{Y}$ ' luminance is sent uncompressed and the $C_{B} C_{R}$ are compressed to save the space. The table.2 below shows the Chroma subsampling methods, color resolution, and luminance and the unused bandwidth. 
Journal of Innovative Image Processing (JIIP) (2019)

Vol.01/ No. 02

Pages: $71-80$

https://www.irojournals.com/iroiip/

DOI: https://doi.org/10.36548/jiip.2019.2.002

\begin{tabular}{|c|c|c|c|c|}
\hline $\begin{array}{l}\text { Chroma sub } \\
\text { sampling } \\
\text { Methods }\end{array}$ & $\begin{array}{c}\text { Color } \\
\text { information }\end{array}$ & Luminance & $\begin{array}{l}\text { Bandwidth } \\
\text { saving \% }\end{array}$ & Description \\
\hline $4: 4: 4$ & $\begin{array}{l}\text { Vertical- } \\
\text { Full } \\
\text { Horizontal - } \\
\text { Full }\end{array}$ & $\begin{array}{l}\text { Vertical - } \\
\text { Full } \\
\text { Horizontal - } \\
\text { Full }\end{array}$ & Zero & No Chroma subsampling \\
\hline $4: 2: 2$ & $\begin{array}{l}\text { Vertical- } \\
\text { Full } \\
\text { Horizontal - } \\
\text { half }\end{array}$ & $\begin{array}{l}\text { Vertical - } \\
\text { Full } \\
\text { Horizontal - } \\
\text { Full }\end{array}$ & 33 & $\begin{array}{l}\text { Horizontal Chroma } \\
\text { resolution is halved }\end{array}$ \\
\hline $4: 2: 0$ & $\begin{array}{l}\text { Vertical- } \\
\text { Half } \\
\text { Horizontal - } \\
\text { Half }\end{array}$ & $\begin{array}{l}\text { Vertical - } \\
\text { Full } \\
\text { Horizontal - } \\
\text { Full }\end{array}$ & 54 & $\begin{array}{l}\text { Both chroma components } \\
\text { ( } \mathrm{Cb} \text { and } \mathrm{Cr} \text { ) are } \\
\text { sampled at half the vertical } \\
\text { resolution of } \mathrm{Y}\end{array}$ \\
\hline $4: 1: 1$ & $\begin{array}{l}\text { Vertical- } \\
\text { Full } \\
\text { Horizontal - } \\
1 / 4\end{array}$ & $\begin{array}{l}\text { Vertical - } \\
\text { Full } \\
\text { Horizontal - } \\
\text { Full }\end{array}$ & 54 & $\begin{array}{l}\text { Both chroma components } \\
\text { ( } \mathrm{Cb} \text { and } \mathrm{Cr} \text { ) are } \\
\text { sampled at one quarter the } \\
\text { horizontal resolution }\end{array}$ \\
\hline
\end{tabular}

Table.2 Chroma Sub Sampling Strategy

(ii) Luminance Masking: this improves the efficiency of the compression in the HDR, this is actually done to reduce the distortions found in the darker and the brighter side of the content. The Weber-Fechner's law states the masking phenomenon as brighter the background luminance higher will be the perceivable visual stimulus difference. The spatial frequency masking and the luminance masking are interrelated. The HDR is integrated with the JND profile to mask the frequencies that are obtained from the quantization matrices.

\section{RESULTS EVALUATION}


Journal of Innovative Image Processing (JIIP) (2019)

Vol.01/ No. 02

Pages: $71-80$

https://www.irojournals.com/iroiip/

DOI: https://doi.org/10.36548/jiip.2019.2.002

The quality of the proposed method is assessed for the HDR encoding compression HDR-VQM metrics in terms of the bit rate savings, and the mean opinion score and compared with the HDR10 and the HDRHLG. The table.3 below provides the quality assessment of the proposed method with the HDR10 and the HDR-HLG

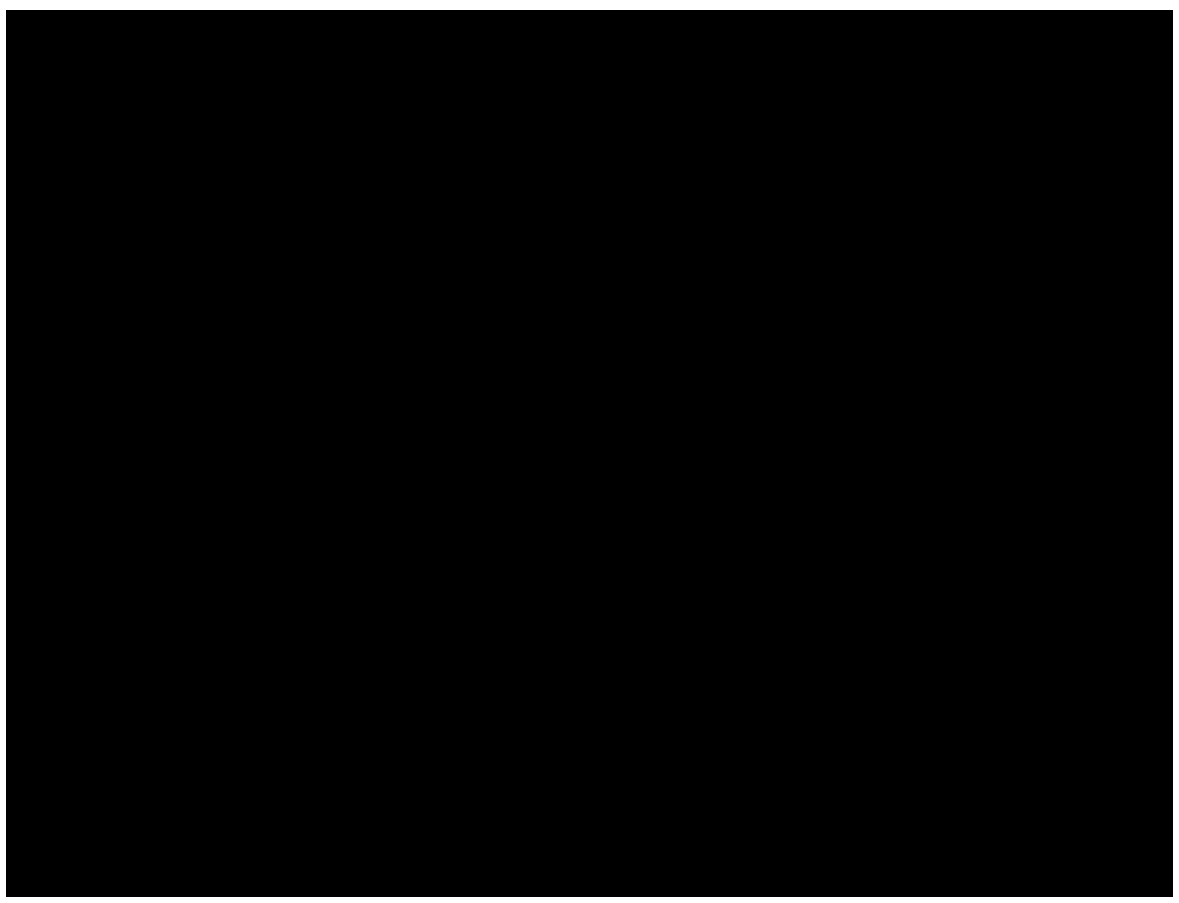

Table.3 The Quality Assessment

The assessment results show that the proposed method improves the quality of the HDR distribution compared to the other methods. In future the paper is to analyze the importance of the HDR encoding, compression in the Computer vision based applications [19-22]

\section{CONLCLUSION}


Journal of Innovative Image Processing (JIIP) (2019)

Vol.01/ No. 02

Pages: $71-80$

https://www.irojournals.com/iroiip/

DOI: https://doi.org/10.36548/jiip.2019.2.002

The paper provides the novel method of two layered backward compatible video encoding methods accompanied by the Chroma subsampling and the luminance masking to improve the color accuracy and remove the nonvisible portion respectively of the video content. The results obtained shows the performance measurement of the novel method HDR video encoding compression (N-HVEC) in term of mean opinion source, bit rate savings and its comparison with the HDR10 and HDR-HLG. This could be utilized further in the applications that necessitate the image classification, and the segmentation process.

\section{References}

[1] Artusi, Alessandro, Rafał K. Mantiuk, Thomas Richter, Philippe Hanhart, Pavel Korshunov, Massimiliano Agostinelli, Arkady Ten, and Touradj Ebrahimi. "Overview and evaluation of the JPEG XT HDR image compression standard." Journal of Real-Time Image Processing 16, no. 2 (2019): 413428.

[2] Roimela, Kimmo, Tomi Aarnio, and Joonas Itäranta. "High dynamic range texture compression." ACM Transactions on Graphics (TOG) 25, no. 3 (2006): 707-712.

[3] Salvucci, Emanuele. "Multi-compatible low and high dynamic range and high bit-depth texture and video encoding system." U.S. Patent 8,237,865, issued August 7, 2012.

[4] Zhang, Yang, Matteo Naccari, Dimitris Agrafiotis, Marta Mrak, and David R. Bull. "High dynamic range video compression exploiting luminance masking." IEEE Transactions on Circuits and Systems for Video Technology 26, no. 5 (2015): 950-964.

[5] Mantiuk, Rafał, Alexander Efremov, Karol Myszkowski, and Hans-Peter Seidel. "Backward compatible high dynamic range MPEG video compression." ACM Transactions on Graphics (TOG) 25, no. 3 (2006): 713-723.

[6] Korshunov, Pavel, and Touradj Ebrahimi. "A JPEG backward-compatible HDR image compression." In Applications of Digital Image Processing XXXV, vol. 8499, p. 84990J. International Society for Optics and Photonics, 2012.

[7] Chalmers, Alan, and Kurt Debattista. "HDR video past, present and future: A perspective." Signal Processing: Image Communication 54 (2017): 49-55.

[8] Lee, Chul, and Chang-Su Kim. "Rate-distortion optimized compression of high dynamic range videos." In 2008 16th European Signal Processing Conference, pp. 1-5. IEEE, 2008.

[9] Blasi, S. G., Matteo Naccari, Rajitha Weerakkody, John Funnell, and Marta Mrak. "The open-source turingcodec: towards fast, flexible and parallel HEVC encoding." (2016): 38-8

[10] https://tech.ebu.ch/docs/groups/beyondhd/HDR_workshop_flyer.pdf. 
Journal of Innovative Image Processing (JIIP) (2019)

Vol.01/ No. 02

Pages: $71-80$

https://www.irojournals.com/iroiip/

DOI: https://doi.org/10.36548/jiip.2019.2.002

[11] Lee, Chul, and Chang-Su Kim. "Rate-distortion optimized compression of high dynamic range videos." In 2008 16th European Signal Processing Conference, pp. 1-5. IEEE, 2008.

[12] Mir, Junaid, Dumidu S. Talagala, Hemantha Kodikara Arachchi, and Anil Fernando. "Adaptive residual mapping for an efficient extension layer coding in two-layer HDR video coding." In 2016 IEEE International Conference on Image Processing (ICIP), pp. 1394-1398. IEEE, 2016.

[13] Mantiuk, Rafał, Alexander Efremov, Karol Myszkowski, and Hans-Peter Seidel. "Backward compatible high dynamic range MPEG video compression." ACM Transactions on Graphics (TOG) 25, no. 3 (2006): 713-723.

[14] Zhang, Yang, Erik Reinhard, and David Bull. "Perception-based high dynamic range video compression with optimal bit-depth transformation." In 2011 18th IEEE international conference on image processing, pp. 1321-1324. IEEE, 2011.

[15] Touzé, David, S. Lasserre, Y. Olivier, R. Boitard, and E. François. "HDR video coding based on local LDR quantization." In HDRi2014-Second International Conference and SME Workshop on HDR imaging. 2014.

[16] Miller, Scott, Mahdi Nezamabadi, and Scott Daly. "Perceptual signal coding for more efficient usage of bit codes." SMPTE Motion Imaging Journal 122, no. 4 (2013): 52-59.

[17] Series, B. T. "High dynamic range television for production and international programme exchange." (2016).

[18] Uhrina, Miroslav, Juraj Bienik, and Tomas Mizdos. "Chroma Subsampling Influence on the Perceived Video Quality for Compressed Sequences in High Resolutions." Advances in Electrical and Electronic Engineering 15, no. 4 (2017): 692-700.

[19] Koresh, Mr H. James Deva. "COMPUTER VISION BASED TRAFFIC SIGN SENSING FOR SMART TRANSPORT." Journal of Innovative Image Processing (JIIP) 1, no. 01 (2019): 11-19.

[20] Raj, Jennifer S. "A COMPREHENSIVE SURVEY ON THE COMPUTATIONAL INTELLIGENCE TECHNIQUES AND ITS APPLICATIONS." Journal of ISMAC 1, no. 03 (2019): 147-159.

[21] Bashar, Abul. "SURVEY ON EVOLVING DEEP LEARNING NEURAL NETWORK ARCHITECTURES." Journal of Artificial Intelligence 1, no. 02 (2019): 73-82.

[22] Joby, P. P. "EXPLORING DEVOPS: CHALLENGES AND BENEFITS." Journal of Information Technology 1, no. 01 (2019): 27-37. 